\title{
Polyarthrite Rhumatoïde en Consultation Rhumatologique à Maradi, Niger
}

\author{
Garba Mahaman Salissou, \\ Service de Rhumatologie Centre Hospitalier Régional de Maradi, \\ Université de Maradi, Niger \\ Condé Kaba, \\ Service de Rhumatologie, CHU Ignace Deen, \\ Université de Conakry, Guinée \\ Garba Idé Adamou, \\ Seydou Alassane, \\ Moussa Fanata, \\ Service de Rhumatologie Centre Hospitalier Régional de Maradi, \\ Université de Maradi, Niger
}

Doi: 10.19044/esj.2019.v15n12p139 URL:http://dx.doi.org/10.19044/esj.2019.v15n12p139

\section{Résumé}

La polyarthrite rhumatoïde (PR) est la plus fréquente des maladies auto-immunes. Rarement décrite au Niger, nous rapportons ses aspects épidémiologiques, cliniques, paracliniques, thérapeutiques et évolutifs en milieu hospitalier. Afin d'analyser ces aspects avec plus de détail une étude prospective a été réalisée sur une période d'un (01) an dans le service de rhumatologie du Centre Hospitalier Régional de Maradi. Nous avons inclus tous les cas de PR en accord avec les critères de classifications de l'ACR/EULAR de 2010. Les données ont été saisies et analysées sur le logiciel SPSS 20.0. Après avoir colligé et étudié 32 observations de PR parmi 846 consultations rhumatologiques, les résultats ont démontré des points divers : l'âge moyen des patients était 38,9 ans, le délai diagnostique était de 6 ans et dans quelques cas il y a eu la présence de manifestations extraarticulaires. Les résultats ont également montré que le syndrome inflammatoire était constant et que la prise en charge reste classique avec des moyens non pharmacologiques, des antalgiques, AINS (agent antiinflammatoire non stéroïdien), infiltrations, et des DMARDs (diseasemodifying anti-rheumatic drug). L'évolution est favorable dans tous les cas. En bref, la polyarthrite rhumatoïde est une réalité au Niger, de mieux en mieux décrite. Le diagnostic est tardif, les manifestations articulaires et systémiques sont les mêmes que chez les caucasiens mais l'auto-immunité 
semble moindre. La maladie est très active, altérant la qualité de vie. En ce qui concerne le traitement, le traitement de fond classique reste efficace.

Mots-clés: Polyarthrite rhumatoïde, Facteur rhumatoïdes, anticorps anti peptides cycliques citrullinés, Niger

\title{
Polyarthrite Rhumatoïde en Consultation Rhumatologique à Maradi, Niger
}

\author{
Garba Mahaman Salissou, \\ Service de Rhumatologie Centre Hospitalier Régional de Maradi, \\ Université de Maradi, Niger \\ Condé Kaba, \\ Service de Rhumatologie, CHU Ignace Deen, \\ Université de Conakry, Guinée \\ Garba Idé Adamou, \\ Seydou Alassane, \\ Moussa Fanata, \\ Service de Rhumatologie Centre Hospitalier Régional de Maradi, \\ Université de Maradi, Niger
}

\begin{abstract}
Rheumatoid arthritis (RA) is the most common auto-immune disease. Rarely described in Niger, we reported its epidemiological, clinical, paraclinical, therapeutic, and evolutionary aspects in hospitals environments. In order to analyze these aspects in detail, a prospective study has been realized over a period of one (1) year in the Rheumatology Department of the Regional Hospital Center of Maradi. We have included all RA cases in accordance with the ACR/EULAR classification criteria of 2010. The data was entered and analyzed on SPSS 20.0 software. After collecting and studying 32 AR observations from among 846 rheumatologic consultations, the results have demonstrated different points: the average patient's age is 38,9 years, the delay for the diagnosis was 6 years, and in some cases extraarticular manifestations have been observed. The results show that the inflammatory syndrome was constant and that the treatment remains classic with non-pharmacologic means, analgesics, NSAID (non-steroidal antiinflammatory agent), infiltrations and DMARDs (disease-modifying anti-
\end{abstract}


rheumatic drug). The evolution is positive in all the cases. Thanks to this study it is possible to affirm that rheumatoid arthritis is a reality in Niger and it has been better described. The diagnosis is late, the articular and systemic manifestations are the same as in Caucasians, but the autoimmunity seems less. The disease is very active, altering the quality of life. As for its treatment, conventional background therapy still remains effective.

Keywords: Rheumatoid arthritis, Rheumatoid factors, antibodies against cyclic citrullinated peptides, Niger

\section{Introduction}

La polyarthrite rhumatoïde (PR) est une maladie auto-immune, fréquente, caractérisée par une inflammation chronique, destructive et déformante des articulations synoviales, de distribution symétrique, distale et acromélique, avec possibilité d'atteinte systémique (Bardin et al., 2015). L'incidence annuelle de la PR dans la population européenne et nordaméricaine est estimée entre 25 et 50/100 000 habitants et la prévalence à 0,5 à 1,\% (Minichiello et al., 2017). En Afrique, la prévalence de la PR varie selon les régions de 0,1 à 0,9\% (Jean del et al., 2002). Au Sénégal elle serait la première maladie systémique dans les services de médecine interne (Kane et al., 2016). Dans le cas du Niger, les études sont rares mais une précédente publication de 2010 à Niamey rapportait les 8 premiers cas documentés, séropositifs (Adehossi et al., 2010). À cet égard, notre travail a pour objectif de déterminer les aspects épidémiologiques, cliniques, paracliniques, thérapeutiques et évolutifs de la polyarthrite rhumatoïde dans le Centre Hospitalier Régional (CHR) de Maradi.

\section{Patients et méthodes}

Nous avons mené une étude prospective, descriptive sur une période d'un (01) an, entre novembre 2017 et octobre 2018 dans le service de rhumatologie du Centre Hospitalier régional (CHR) de Maradi au Niger. Le service a été ouvert en octobre 2017. Il reçoit tous les cas de rhumatismes en consultation ou en hospitalisation au CHR. Nous avons inclus tous les cas de polyarthrite rhumatoïde en accord avec les critères de classifications de l'ACR/EULAR de 2010 (Aletaha et al., 2010). Pour chaque patient, les données suivantes ont été collectées : démographiques (l'âge, le genre), cliniques (les antécédents), les manifestations articulaires (le nombre d'articulations douloureuses et gonflées, les déformations ankylosantes ou non, l'intensité de la douleur par l'EVA (Échelle Visuelle Analogique), la durée d'évolution ( $P R$ très récente $<6$ mois; débutante $<18$ mois ; récente $<$ 5 ans et ancienne $\geq 5$ ans) et extra articulaires (syndrome sec, nodules rhumatoïdes, la splénomégalie, les adénopathies, les signes cardio-vasculaires 
et glandulaires). Au plan biologique, nous avons analysé la numération formule sanguine (NFS), vitesse de sédimentation (VS), C réactive protéine (CRP quantitative et qualitative) et les Facteurs rhumatoïdes (Latex et Waaler Rose). Il faut rappeler qu'au début de notre étude le test n'était pas disponible sur place. Dans le souci d'améliorer notre plateau, le Latex est rendu disponible au sein de l'hôpital et le Latex-Waaler Rose dans un laboratoire privé. Les anticorps anti peptides cycliques citrullinés de type 2 (ACPA) ont aussi été détectés.

Sur l'imagerie, nous avons analysé les données de l'échographie articulaire (synovite grade de Szkudlarek) aussi bien que des radiographies standard des main-poignets (face) selon le score de Steinbroker et la radiographie des pieds (3/4) à la recherche de lésions structurales. L'activité de la maladie a été évaluée à l'aide de l'échelle d'activité de la maladie sur 28 articulations (DAS28-VS). L'état fonctionnel a été évalué à l'aide du Standford Health Assessment Questionnaire (HAQ). Les données ont été analysées avec le logiciel SPSS 20.0. Test statistique $<0,05$.

\section{Résultats}

Nous avons colligé 32 observations de polyarthrite rhumatoïde parmi 846 consultations rhumatologiques soit une proportion hospitalière globale de $3,7 \%$. Il s'agissait de 8 hommes $(25 \%)$ et 24 femmes $(75 \%)$ avec un Sex-ratio de 0,33 . L'âge moyen au début apparent de la maladie était de 38,9 $\pm 16,4$ ans [12 - 75 ans]. Le délai diagnostic était de 6 ans [1 mois - 30 ans] (Tableau I).

Sur le plan clinique, nos patients avaient tous une notion de polyarthrite symétrique et présentaient à l'admission en moyenne 15 articulations douloureuses [2 - 28] et au moins 5 articulations gonflées [0 - 18]. Les déformations majoritairement retrouvés étaient des doigts en boutonnières, des mains en coup de vent cubital et en dos de chameau dans respectivement huit cas $(25 \%)$ ainsi que six cas $(18,8 \%)$ de pouce en $Z$, un cas $(3,1 \%)$ de col de cygne et un cas $(3,1 \%)$ des mains en maillet. Au plan évolutif, la PR est jugée très récente dans six cas $(19,3 \%)$, débutante dans huit cas $(26 \%)$, récente dans six cas $(19,3 \%)$, ancienne et/ou séquellaire dans 11 cas $(35,4)$.

Sur le plan extra-articulaire (9 cas/32), la polyarthrite s'est compliquée d'un syndrome de Sjögren secondaire dans sept cas $(23,9 \%)$. Les nodules rhumatoïdes étaient présents chez un patient $(3,1 \%)$ ainsi qu'un cas de syndrome de Felty (3,1\%), La PR s'est compliquée d'une pneumopathie interstitielle diffuse (PID) dans deux cas $(6,25 \%)$. Sur le plan cardiovasculaire, deux cas (6,25\%) d'hypertension artérielle étaient notés. Sur le plan endocrino-métabolique, un goitre non exploré était noté ainsi que trois cas de fracture vertébrale ostéoporotique. Un cas de portage chronique de l'hépatite B était noté. 
Tableau I. Répartition des 32 patients selon l'âge moyen, le délai diagnostic, les atteintes articulaires et la biologie

\begin{tabular}{|c|c|c|c|}
\hline & Genre & Moyenne & Extrêmes \\
\hline \multirow[t]{3}{*}{ Age moyen } & & $38,9 \pm 16,4$ & {$[12-75]$} \\
\hline & Masculin & $48,35 \pm 20,4$ & {$[19-75]$} \\
\hline & Féminin & $36,15 \pm 14,4$ & {$[12-70$} \\
\hline \multirow[t]{3}{*}{ Délai diagnostic moyen } & & 6 ans & [1 mois - 30 ans] \\
\hline & Masculin & 2,6 ans & [3 mois -8 ans] \\
\hline & Féminin & 6,88 ans & [1 mois -30 ans] \\
\hline \multirow[t]{3}{*}{$\begin{array}{l}\text { Nombre d'articulations } \\
\text { douloureuses }\end{array}$} & & 15 & {$[2-28]$} \\
\hline & Masculin & 12 & {$[3-21]$} \\
\hline & Féminin & 16 & {$[2-28]$} \\
\hline \multirow[t]{3}{*}{$\begin{array}{l}\text { Nombre } \quad \text { d'articulations } \\
\text { gonflées }\end{array}$} & & 5 & {$[0-18]$} \\
\hline & Masculin & 4 & {$[2-8]$} \\
\hline & Féminin & 5 & {$[0-18]$} \\
\hline Biologie & Effectif $(\%)$ & & \\
\hline VS élevée & $31 / 32(98,8)$ & $91,86 \pm 37,2$ & [22 - 160] \\
\hline CRP positive & $10 / 10(100)$ & & \\
\hline FR positif & $9 / 25(36)$ & & \\
\hline ACPA positif & $3 / 7(42,9)$ & 197,5 & {$[54-341]$} \\
\hline
\end{tabular}

Sur le plan biologique, le syndrome inflammatoire était constant avec une vitesse de sédimentation accélérée dans 31 cas avec un taux moyen de $91,86 \pm 37,2$ [22-160] et une C réactive protéine augmentée dans tous les 10 examens réalisés. Les facteurs rhumatoïdes étaient présents chez 9 patients sur 25 soit $36 \%$ et les anticorps anti CCP présents chez 3 patients sur 7 soit $42,9 \%$ avec un taux moyen de 197,5 U/ml (Tableau I).

Sur le plan radiologique, chez trois patients bénéficiaires, l'échographie montrait un pannus (Grade 3 de Szkudlarek) et des érosions osseuses. Les radiographies standards chez 30 patients, étaient évaluées selon le score de Steinbroker. Le stade I a été retrouvé chez 7 (23,3\%) patients, les stades II et III chez respectivement 6 patients (20\%) et le stade IV chez 11 $(36,7 \%)$.

Sur le plan thérapeutique, tous les patients avaient bénéficié d'un traitement non pharmacologique (information, éducation, communication) mais aussi symptomatique contre la douleur. Six patients $(18,75 \%)$ avaient 
bénéficié d'infiltration complémentaire. Les corticoïdes étaient prescrits chez 31 patients $(96,8 \%)$ dont quatre cas en mini-bolus et les antipaludéens de synthèses chez 21 patients $(65,6 \%)$. Le traitement immunosuppresseur a été appliqué en 2 cas sous salazopyrine (6,25\%), 2 cas sous azathioprine $(6,25 \%)$, et 30 cas sous méthotrexate $(93,75 \%)$.

L'activité de la maladie était jugée modérée chez neuf patients $(37,5 \%)$ et forte chez 15 patients $(62,5 \%)$ avec un DAS28-vs moyen de 5,55 $\pm 1,02$ à l'admission. Le retentissement fonctionnel évalué par le Standford Health Assessment Questionnaire (HAQ) montrait une nette altération de la qualité de vie. À l'admission l'HAQ était en moyenne de 2,38 $\pm 0,54$ [0,625 - 2,875] et à 6 mois de traitement à $0,64 \pm 0,58[0,125-2,5]$. Une corrélation significative a été notée entre les valeurs d'HAQ à l'admission et sous traitement $\left(\mathrm{p} 0,021^{*}\right)$ (Tableau II). Aucun cas de décès n'a été noté.

Tableau II. Répartition selon l'activité de la maladie à l'admission et le retentissement

\begin{tabular}{|c|c|c|c|c|}
\hline DAS28 -vs (Activité) & Effectifs (\%) & Moyenne & Extrêmes & \\
\hline Légère & 0 & & & \\
\hline Modérée & $9(37,5)$ & & & \\
\hline Forte & $15(62,5)$ & & & \\
\hline Total & 24 & $5,55 \pm 1,02$ & {$[3,77-7,72]$} & \\
\hline \multicolumn{5}{|l|}{$H A Q$} \\
\hline HAQ de base & 30 & $2,38 \pm 0,54$ & {$[0,625-2,875]$} & \\
\hline HAQ à 6 mois & 30 & $0,64 \pm 0,58$ & {$[0,125-2,5]$} & p $0,021^{*}$ \\
\hline
\end{tabular}

\section{Discussion}

La polyarthrite rhumatoïde est le rhumatisme inflammatoire le plus fréquent surtout chez la femme. L'incidence annuelle de la PR dans la population européenne et nord-américaine est estimée entre 25 et 50/100 000 habitants et la prévalence à 0,5 à 1,0 \% (Minichiello et al., 2017). Elle était initialement considérée comme rare en Afrique, avec 0,3\% de prévalence en Afrique du sud (Jeandel et al., 2002 ; Mijiyawa, 1995) ; cependant les études récentes estiment cette prévalence superposable à celle occidentale surtout en zone urbaine, entre 0,1 et $0,9 \%$ en Afrique de l'Est et du Sud, entre 0,1 à 0,5 $\%$ en Afrique de l'Ouest (Jeandel et al., 2002 ; Mijiyawa, 1995 ; Roux, 2002). Notre proportion de $3,7 \%$ est hospitalière et ne peut donc pas être comparée avec celle occidentale faite sur la population générale. Néanmoins elle précise que cette pathologie existe bien au Niger avec une incidence croissante. Cette incidence est probablement sous-estimée en rapport avec la méconnaissance de la pathologie par certains praticiens et la création toute récente du service de rhumatologie ( 1 an). Cependant Houzou et al, au Togo au cours d'une étude panoramique des arthropathies inflammatoires ont rapporté 62 cas à 
Lomé Tokoin (Houzou et al., 2013) et 11 cas au CHU de Kara (Houzou et al., 2018).

La maladie atteint surtout la femme dans 75 à $80 \%$ des cas (Bardin et al., 2015). Ferreira et al, trouvaient 79,1\% de femmes (Ferreira et al., 2017) alors que Farzy et al, ont trouvé $90 \%$ de femmes (Harzy et al., 2007). Ces données sont comparables avec nos résultats avec $75 \%$ de femmes. L'âge moyen au début apparent de la maladie chez nos patients est de 38,9 ans avec des différences selon le genre. Chez la femme le début semble plus précoce (36,15 ans) par rapport aux hommes (48,35 ans). Nos données sont comparables avec celles d'Adehossi et al, au Niger avec 38 ans (Adehossi et al., 2010), Ntsiba, au Congo avec 41 ans d'âge moyen (Ntsiba et al., 2007). Cette précocité d'apparition chez les africains est déjà décrite dans la littérature (Roux, 2002) probablement liée au rôle des hormones (œstrogènes) dans la pathogénie ainsi que l'implication des facteurs génétiques de susceptibilité.

Le mode de transmission est difficile à établir chez nos patients du fait de l'insuffisance du plateau technique ne permettant pas une étude génétique. Toute fois un lien génétique est établi chez les caucasiens entre la PR et HLA DR1, DR4 et DR10 (Bardin et al., 2015). Une étude sénégalaise a trouvé un lien entre PR et HLA DR10 (Dieye et al., 1997). Une autre étude a trouvé un lien avec PR et HLA-DR1 en Afrique de l'Ouest (Roux, 2002), correspondant à notre zone géographique. Par contre lors d'une enquête sur la présence des loci de susceptibilités caucasiens chez les africains (Camerounais et Nigérians), il a été trouvé une rareté des polymorphismes non HLA notamment PTPN22, IL2RA, FCGR2A et IL2 / IL21, moins de 2\%. Le risque génétique global, n'a pas prédit la survenue de PR chez les africains contrairement aux britanniques, suggérant une différence génétique de susceptibilité entre ces populations (Viatte et al., 2012).

Les atteintes articulaires de nos patients sont les mêmes décrites dans la littérature africaine (Ntsiba et al., 2007). Dans 76,7\% des cas, nos patients avaient des atteintes structurales. Nos données sont comparables à celles de la littérature malgré la bénignité décrite en Afrique de l'Ouest [7]. Ntsiba H, au Congo rapportait 12 patients $(24 \%)$ au stade 4 de Steinbrocker, 13 au stade $3(26 \%), 20$ au stade $2(40 \%)$ et 5 au stade 1 (10\%) (Ntsiba et al., 2007) et Adehossi E rapportait les mêmes type de lésions avec une carpite dans 37,5\% des cas (Adehossi et al., 2010).

Le syndrome de Sjögren complique la PR dans 4 à $31 \%$ des cas (Bardin et al., 2015). Nos résultats sont comparables avec 7 cas $(23,9 \%)$ dont 1 cas avec des ACPA. Ntsiba, rapportait 4 cas (8\%) (Ntsiba et al., 2007).

Un patient $(3,12 \%)$ présentait des nodules rhumatoïdes en dessous des olécranes associés à une activité forte, persistante et la présence d'ACPA. Adhehossi rapportait 2 cas sur 8 (Adehossi et al., 2010) alors qu'au Sénégal 
et au Congo, Diallo $\mathrm{S}$ et al et Ntsiba $\mathrm{H}$ et al ont trouvé respectivement $1,1 \%$ et 6\% (Diallo et al., 2001; Ntsiba et al., 2007).

Le taux de PID est passé de $5 \%$ à $20-30 \%$ depuis l'avènement de la tomodensitométrie (Bardin et al., 2015). Nous rapportons deux cas de PID $(6,25 \%)$ de même qu' Adefuye, au Nigeri, deux cas (Adefuye et al., 2014), comparable à celle de Kakpovi au Togo 6,5\% (Kakpovi et al., 2017), ainsi qu'à celle de Roux $\mathrm{H}$ et al en Afrique du Sud qui ont trouvé 4,8\% (Roux, 2002).

Nous rapportons également un cas de syndrome de Felty $(3,1 \%)$, comparables aux deux cas (4\%), rapportés par Ntsiba (Ntsiba et al., 2007).

La PR est facteur de risque d'ostéoporose indépendant de la corticothérapie. El Maghraoui, rapportait 36\% de fracture vertébrale (El Maghraoui et al., 2010). Notre taux de $12,5 \%$ (3 cas $/ 24$ femmes) est probablement sous-estimé $\mathrm{du}$ fait de la non disponibilité de l'ostéodensitométrie.

Les facteurs rhumatoïdes sont classiquement retrouvés chez 60 à $80 \%$ des patients selon la technique de recherche. Cependant, nous rapportons $36 \%$ (Latex et Waaler Rose). Nos données sont comparables à celles retrouvés au Zimbabwe 37\% (Davis et al., 1989), au Niger 48\% (Adebajo et al., 1992) mais moindre qu'au Congo 60\% (Ntsiba et al., 2007) et au Burkina 70\% (Ouédraogo et al., 2011). Ces anticorps semblent être moins présents chez certains africains (Mijiyawa et al., 1995 ; Roux, 2002). Cette séronégativité est peut être due à des « facteurs rhumatoïdes cachés » (Viatte et al., 2012) mais aussi et surtout aux particularités génétiques et environnementales, notamment la rareté des loci de susceptibilité caucasiens chez certains africains (Mijiyawa et al., 1995 ; Viatte et al., 2012).

Les anticorps anti peptides citrullinés (ACPA) sont classiquement retrouvés dans environ $80 \%$ des PR (Anzilotti et al., 2010). Nous rapportons trois patients positifs sur sept $(42,9 \%)$. Ce faible taux de réalisation est dû au fait que la recherche de ces anticorps n'est pas disponible sur place mais les prélèvements sont acheminés en France avec des coûts inaccessibles pour nos populations pauvres.

L'activité de la maladie évaluée par le DAS 28 montrait qu'à l'admission $37,5 \%$ des patients avaient une activité modérée et $62,5 \%$ une activité forte. La valeur moyenne était de 5,5 en rapport avec le grand nombre d'articulations douloureuses et gonflées mais aussi de l'intensité du syndrome inflammatoire. Cette constance du syndrome inflammatoire était rapportée par Adéhossi, avec une VS accélérée dans tous les cas (Adehossi et al., 2010). Khawaja, trouvait un DAS 28 de départ de 6,5 (Kwawadja et al., 2017) alors que Ndongo S, au Sénégal, trouvait un DAS 28 de 6,8 (Ndongo et al., 2009).

En ce qui concerne la prise en charge, nos patients ont tous bénéficié d'un traitement non pharmacologique. La prise en charge thérapeutique 
combinait des moyens symptomatiques contre la douleur, des infiltrations, un traitement de fond classique et des moyens physiques. Elle répondait aux recommandations en vigueur (Gaujoux-Viala et al., 2014). Malgré l'absence de biothérapie, l'activité de la maladie s'est réduite et la qualité de vie s'est nettement améliorée. Ainsi, la qualité de vie mesurée par HAQ à 2,38 lors

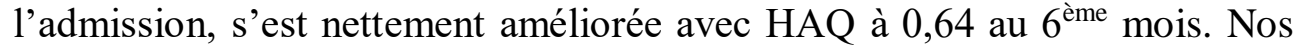
données sont comparables à celles de Volet et al qui trouvait HAQ de 0,5 après 6 mois de traitement par tofacitinib (Strand et al., 2018). L'évolution est jusque -là favorable dans tous les cas.

\section{Conclusion}

La polyarthrite rhumatoïde est une réalité dans nos régions. Son incidence est certainement croissante en rapport avec la présence de spécialistes internistes et rhumatologues dans les hôpitaux régionaux. Le diagnostic est tardif mais les manifestations cliniques sont les mêmes. L'autoimmunité semble moindre chez nos patients. L'amélioration future du plateau technique nous facilitera la recherche de ces anticorps jusque-là inaccessibles pour la majorité de nos patients. La prise en charge reste classique mais efficace avec une évolution favorable.

Déclaration de liens d'intérêts : Les auteurs déclarent ne pas avoir de liens d'intérêts.

\section{References:}

1. Adebajo, AO., Charles, PJ., Hazleman, BL., \& Maini, RN. (1992). Serological profile of rheumatoid arthritis in West Africa. Rheumatology International;12(6):235-8.

2. Adefuye, BO., Adelowo, OO., Adefuye, PO., \& Oguntona, OO. (2014). RHEUMATOID ARTHRITIS ASSOCIATED WITH PULMONARY FIBROSIS IN NIGERIANS: TWO CASE REPORTS. East Afr Med J. sept;91(9):323-5.

3. Adehossi, E., Landais, C., Souna, BS., Guida, S., Gbaguidi, F., Toure AI, et al. (2010). Premiers cas documentés de polyarthrite rhumatoïde au Niger. Cahiers de Santé. janv;(1):41-45.

4. Aletaha, D., Neogi, T., Silman, AJ., Funovits, J., Felson, DT et al. (2010). Rheumatoid arthritis classification criteria: an American College of Rheumatology/European League Against Rheumatism collaborative initiative. Arthritis Rheum. Sep;62(9):2569-81

5. Anzilotti, C., Pratesi, F., Tommasi, C., \& Migliorini, P. (2010). Peptidylarginine deiminase 4 and citrullination in health and disease. Autoimmunity Reviews. janv;9(3):158-60. 
6. Bardin T, Richette P, Dieudé P et al. Manifestations systémiques de la polyarthrite rhumatoïde. In : Guillevin L, Meyer O, Hachulla E, Sibilia J. Traité des maladies et syndromes systémiques. 6eme ed. Paris : Flammarion Médecine-Sciences ;.p 434-89.

7. Davis, P., Stein, M., Ley, H., \& Johnston, C. (2015). Serological profiles in the connective tissue diseases in Zimbabwean patients. Annals of the Rheumatic Diseases. 1 janv 1989;48(1):73-6.

8. Dieye, A., Diallo, S., Diatta, M., Thiam, A., Ndiaye, R., Bao, O et al. (1997). [Identification of HLA-DR alleles for susceptibility to rheumatoid polyarthritis in Senegal]. Dakar Med.;42(2):111-3.

9. Diallo, S., Pouye, A., Dangon, JM., Toure, S., Ka, MM., N'Diaye, R et al. (2001). Syndrome de Gougerot-Sjögren Chez le Noir africain. Étude prospective de 130 observations [résumé]. Rev Rhum[Ed Fr]; 68 : 1009.

10. El Maghraoui, A., Rezqi, A., Mounach, A., Achemlal, L., Bezza, A., \& Ghozlani, I. (2010). Prevalence and risk factors of vertebral fractures in women with rheumatoid arthritis using vertebral fracture assessment. Rheumatology. 1 juill;49(7):1303-10.

11. Ferreira, RJO., Dougados, M., Kirwan, JR., Duarte, C., de Wit, M., Soubrier, M et al. (2017). Drivers of patient global assessment in patients with rheumatoid arthritis who are close to remission: an analysis of 1588 patients. Rheumatology. 1 sept;56(9):1573-8.

12. Gaujoux-Viala, C., Gossec, L., Cantagrel, A., Dougados, M., Fautrel, B., Mariette, X et al. (2014). Recommandations de la Société française de rhumatologie pour la prise en charge de la polyarthrite rhumatoïde. Revue du Rhumatisme. juill;81(4):303-12.

13. Harzy, T., Allali, F., Bennani-Othmani, M., \& Hajjaj-Hassouni, N. (2007). [Radiological characteristics of the lumbar spine in patients with rheumatoid arthritis]. Presse Med. oct;36(10 Pt 1):1385-9.

14. Houzou, P., Fianyo, E., Kakpovi, K., Koffi-Tessio, VS., Tagbor, KC., Oniankitan, O, et al. (2018). Panorama of inflammatory arthropathies in rheumatologic consultations in Northern Togo. Med Sante Trop. 1 août;28(3):320-3.

15. Houzou, P., Oniankitan, O., Kakpovi, K., Koffi-Tessio, VES., Tagbor, KC., Fianyo, E et al. (2013). [Rheumatic diseases profile of 13517 West African patients]. Tunis Med. janv;91(1):16-20.

16. Jeandel, P. \& Roux, H. (2002). Épidémiologie des affections rhumatologiques en Afrique subsaharienne. Revue du Rhumatisme. sept 2002;69(8):764-76.

17. Kalpovi, K., Koffi-Tessio, V., Houzou, P., Fianyo, E., Kolou, M., Kuéviakoé, M.I. et al. (2017). Profil de la polyarthrite rhumatoïde en 
consultation rhumatologique à Lomé (Togo). European Scientific Journal May 2017; 13(15) : 1857 - 7881.

18. Kane, BS., Ndongo, S., Ndiaye, AA., Djiba, B., Niasse, M., Diack, N et al. (2016). Maladies systémiques en médecine interne «contexte africain »: aspects épidémiologiques et classification. La Revue de Médecine Interne. juin 2016;37:A37.

19. Khawaja, MN., Bergman, MJ., Yourish, J., Pei, J., Reiss, W., \& Keystone, E. (2017). Routine Assessment of Patient Index Data 3 and the American College of Rheumatology/European League Against Rheumatism Provisional Remission Definitions as Predictors of Radiographic Outcome in a Rheumatoid Arthritis Clinical Trial With Tocilizumab: Predicting Future Good Radiographic and Functional Outcomes. Arthritis Care \& Research. mai;69(5):609-15.

20. Mijiyawa, M. (1995). Epidemiology and semiology of rheumatoid arthritis in Third World countries. Rev Rhum Engl Ed. févr;62(2):121-6.

21. Minichiello, E., Semerano, L. \& Boissier, M-C. (2017). Incidence, prévalence et sévérité de la polyarthrite rhumatoïde au XXI e siècle. Revue du Rhumatisme Monographies. sept;84(4):303-10.

22. Ndongo, S., Lekpa, FK., Ka, MM., Ndiaye, N., \& Diop, TM. (2009). Presentation and severity of rheumatoid arthritis at diagnosis in Senegal. Rheumatology. 1 sept;48(9):1111-3.

23. Ntsiba, H., Biléckot, R., \& Bissombolo, R. (2007). La polyarthrite rhumatoïde au Congo-Brazzaville. Revue du Rhumatisme. 1 nov;74(10):1042-3.

24. Ouédraogo, D-D., Singbo, J., Diallo, O., Sawadogo, SA., Tiéno, H., \& Drabo, YJ. (2011). Rheumatoid arthritis in Burkina Faso: clinical and serological profiles. Clinical Rheumatology. déc;30(12):1617-21.

25. Roux, H. (2002). Polyarthrite rhumatoïde en Afrique subsaharienne. Revue du Rhumatisme. sept; 69(8):797-800.

26. Strand, V., Lee, EB., Yazici, Y., Dikranian, A., Wilkinson, B., Takiya, L et al. (2018). Evaluation of disease activity in patients with rheumatoid arthritis treated with tofacitinib by RAPID3: post hoc analyses from two phase 3 trials. Clin Rheumatol. août;37(8):2043-53

27. Viatte, S., Flynn, E., Lunt, M., Barnes, J., Singwe-Ngandeu, M., Bas, $S$ et al. (2012). Investigation of Caucasian rheumatoid arthritis susceptibility loci in African patients with the same disease. Arthritis Research \& Therapy;14(6):R239. 